\title{
The effect of dust obscuration in RR Telescopii on optical and IR long-term photometry and Fe II emission lines ${ }^{\star}$
}

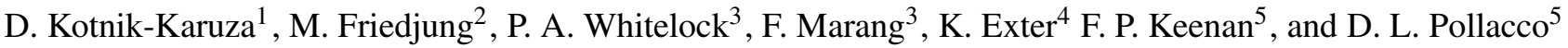 \\ 1 Physics Department, Faculty of Arts and Sciences, Omladinska 14, 51000 Rijeka, Croatia \\ e-mail: dubravka.karuza-kotnik@ri.htnet.hr \\ 2 Institut d'Astrophysique de Paris UMR7075 CNRS, Université Pierre \& Marie Curie, 98 bis Boulevard Arago, 75014 Paris, France \\ e-mail: friedjung@iap.fr \\ 3 South African Astronomical Observatory, Observatory, South Africa and national Astrophysics and Space Science Programme, \\ University of Cape Town, 7701 Rondebosch, South Africa \\ e-mail: paw@saao.ac.za \\ 4 Instituto de Astrofisica de Canarias, La Laguna (Tenerife), Spain \\ e-mail: katrina@iac.es \\ 5 Dept. of pure and applied physics, Queens University, Belfast, Northern Ireland \\ e-mail: F.Keenan@qub.ac.uk
}

Received 12 September 2005 / Accepted 19 January 2006

\begin{abstract}
Aims. Infrared and optical photometric and spectroscopic observations of the symbiotic nova RR Tel are used to study the effects and properties of dust in symbiotic binaries containing a cool Mira component, as well as showing "obscuration events" of increased absorption, which are typical for such Miras.

Methods. A set of photometric observations of the symbiotic nova RR Tel in different wavelength bands - visual from 1949 to 2002 and near-infrared $(J H K L)$ from 1975 to 2002 - are presented. The variability due to the normal Mira pulsation was removed from the $J H K L$ data, which were then compared with the American Association of Variable Star Observers' (AAVSO) visual light curve. The changes of the Fe II emission line fluxes during the 1996-2000 obscuration episode were studied in the optical spectra taken with the Anglo-Australian telescope.

Results. We discuss the three periods during which the Mira component was heavily obscured by dust as observed in the different wavelength bands. A change in the correlations of $J$ with other infrared magnitudes was observed with the colour becoming redder after JD 2446600 . Generally, $J-K$ was comparable, while $K-L$ was larger than typical values for single Miras. A distance estimate of $2.5 \mathrm{kpc}$, based on the IR data, is given. A larger flux decrease for the permitted than for the forbidden Fe II lines, during the obscuration episode studied, has been found. There is no evidence for other correlations with line properties, in particular with wavelength, which suggests obscuration due to separate optically thick clouds in the outer layers.
\end{abstract}

Conclusions.

Key words. stars: binaries: symbiotic - stars: individual: RR Tel - stars: circumstellar matter

\section{Introduction}

The symbiotic nova RR Tel is an interacting binary, consisting of a Mira-type cool component, a hot component thought to be a white dwarf, an extended nebular envelope and a dust envelope around the cool component. Since its nova-like optical outburst in 1944 long term spectroscopic and photometric observations have been carried out in the UV, optical and IR spectral regions in order to establish a satisfactory model of this puzzling object. Inter-comparison of data from observations in different wavelength regions made a significant contribution to these attempts (Heck \& Manfroid 1985; Contini \& Formiggini 1999; Penston et al. 1983; Whitelock 1987).

RR Tel belongs to the class of symbiotic Miras, where the cool giant is a Mira variable (Whitelock 2003). Normal single Mira variables are pulsating stars at the top of the asymptotic giant branch (AGB) with periods in the 100 to more than

* Table 1 is also available in electronic form at the CDS via anonymous ftp to cdsarc.u-strasbg.fr $(130.79 .128 .5)$ or via http://cdsweb.u-strasbg.fr/cgi-bin/qcat?]/A+A/452/503
2000 days range. They have strong winds, which are thought to be first levitated by pulsations, and then accelerated by the action of radiation pressure on the abundant dust, which will then drag the gas. The dust can also produce "obscuration events" of increased absorption, which are rare in single oxygen-rich Miras, but which occur for the cool components of most symbiotic Miras. Mira variables can be either oxygen- or carbon-rich, RR Tel being a member of the oxygen-rich class.

From the evolutionary point of view a low or intermediate mass star will evolve along the AGB before it becomes a white dwarf. Symbiotic Miras, unlike other symbiotic binaries, have large separations and corresponding long orbital periods of probably not less than 20 years, known orbital periods of other symbiotic binaries being usually less than 4 years (Belczynski et al. 2000). However, the winds are so strong, that accretion by the compact component can still be high, in spite of the large separation. An accreting white dwarf can then undergo continuous thermonuclear burning of the accreted material or occasional epochs of such burning. The latter, sporadic thermonuclear events, are thought to explain the nova like outbursts. 
In our present work, we have reanalyzed infrared and optical photometry, as well as emission line fluxes. As we wish in particular to understand better the obscuration events, we used the infrared photometry of RR Tel and compared it with that of normal isolated Miras (Whitelock et al. 1994, 2000; Le Bertre 1993; Smith 2003) as well as with Miras accompanied by hot components in symbiotics (Whitelock 1987, 1988, 2003).

Infrared photometry of RR Tel was published by Feast et al. (1983), Feast et al. (1977), Whitelock (1987) and Whitelock (1998). The $J$ magnitudes up to 2002 were illustrated in Whitelock (2003). Here we use the whole available dataset to construct the JHKL light curves and compare them with the visual light curve obtained for the same epochs. The colour changes of RR Tel up to 2002 have also been investigated.

In this work we also show the visual light curve of RR Tel covering the time interval 1949-2002. The light curve of Contini and Formiggini (1999), taken from different sources, ends in 1995. Recent observations, from 1995 on, are also interesting because there is evidence of a systematic decrease of the optical line fluxes from 1996 to 2000.

Such behaviour was observed for the Fe II lines (Kotnik-Karuza et al. 2003) as well as for the fluxes of other ions measured in the optical spectra during the period mentioned (Kotnik-Karuza et al. 2004a,b). No evidence of fading was found (Kotnik-Karuza et al. 2002) between the July 1993 observations described by McKenna et al. (1997) and the July/August 1996 observations described by Crawford et al. (1999). We do not have information, which could enable us to make a comparison in other wavelength regions.

Searching for a possible relationship between the IR fading and the spectroscopic changes of the hot source, we concentrate here on the Fe II and [FeII] fluxes, less susceptible than fluxes of lines of more ionized species formed nearer the hot component, to be affected by changes of the temperature of the hot component (Mürset \& Nussbaumer 1994).

The distance of RR Tel is not well defined, estimates of it ranging between about $2.5 \mathrm{kpc}$ (Thackeray 1977; Penston et al. 1983 ) to $3.6 \mathrm{kpc}$ (Feast et al. 1983). By use of more recent material and with the considerably increased amount of observational IR data for RR Tel and other symbiotic Miras, we give a revised distance estimate for RR Tel.

\section{Observations and methods of analysis}

The visual magnitudes of RR Tel were measured by the AAVSO observers from the Southern Hemisphere between the years 1949 and 2002. Figure 1 shows the smoothed visual light curve which was obtained by taking means of magnitudes over 30 day intervals.

The JHKL magnitudes monitored from the South African Astronomical Observatory (SAAO) from 1975 to 2002 are given in Table 1. Infrared JHKL light curves of RR Tel are affected by Mira pulsations with a period of 387 days (Feast et al. 1983) and by long-term variations as observed in many symbiotic Miras (Whitelock 1987; Mikolajewska et al. 1999; Whitelock 2003). In order to isolate the long-term trends, we have corrected the light curves for Mira pulsations by a procedure in which the observations were binned at the same Mira phase, taking means of the magnitudes in each IR band over a tenth of the Mira period. The points were plotted separately at the same phase within each of these bins. Then we superposed the curves at different phases by shifting them vertically with respect to the curve with the best distribution of observations to obtain a minimum deviation by a least squares fit. The resulting $J H K L$ curves corrected for

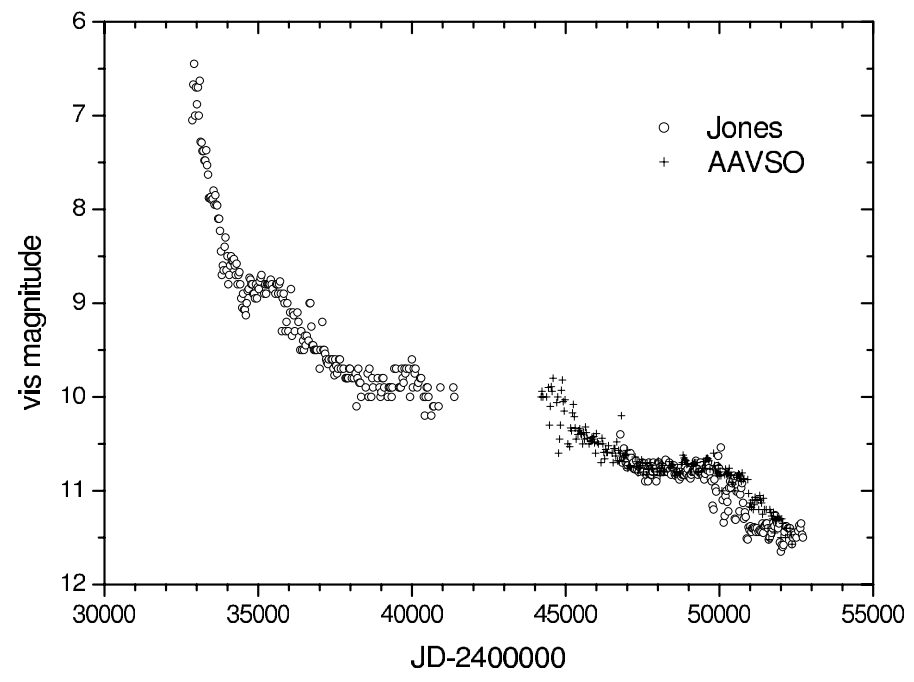

Fig. 1. RR Tel averaged visual estimates over 30 days for the years 1949-2002. Crosses refer to AAVSO observational data, collected from several observers and given to us by the late J. Mattei. Circles correspond to the observations of Albert Jones.

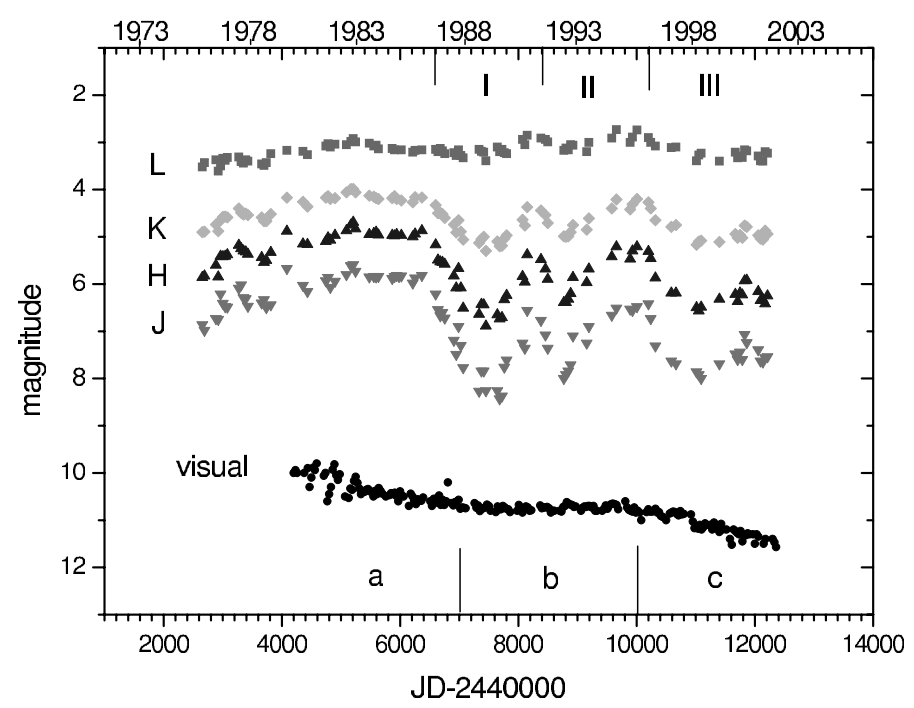

Fig. 2. Binned IR and visual light curves from 1975 to 2002. The epochs I, II and III refer to three obscuration events, while a, b and c mark different segments of the visual light curve (see text).

Mira pulsations are shown in Fig. 2 together with the overlapping part of the visual light curve for comparison. Let us note that the $J H K L$ magnitudes are accurate to better than $0.03 \mathrm{mag}$ in $J H K$ and $0.05 \mathrm{mag}$ at $L$. Some of the early data, which had been published in Feast et al. (1983), were subsequently slightly corrected to the SAAO system as defined by Carter (1990).

In addition, flux calibrated optical spectra, taken with the Anglo-Australian telescope in 1996 and 2000, are compared. The former described by Crawford et al. (1999), covering the region from 3100-9800 $\AA$, was obtained on July 22, 1996 with a resolution of about 50000 and was flux calibrated on August 2, 1996. The latter was taken in July 2000 with almost twice the spectral resolution and was flux calibrated with two other spectra, including one taken with the HST in October 2000. 
Table 1. JHKL magnitudes of RR Tel from 1975 to 2002.

\begin{tabular}{|c|c|c|c|c|c|c|c|c|c|c|c|c|c|c|}
\hline JD-2 440000 & $J$ & $H$ & $K$ & $\bar{L}$ & JD-2440000 & $J$ & $H$ & $K$ & $\bar{L}$ & JD-2440000 & $\bar{J}$ & $H$ & $K$ & $\bar{L}$ \\
\hline 2653.50 & 5.84 & 4.94 & 4.21 & 3.17 & 5215.34 & 5.590 & 4.688 & 3.988 & 2.919 & 8067.60 & 6.225 & 4.918 & 3.950 & 2.590 \\
\hline 2689.50 & 6.15 & 5.10 & 4.38 & 3.29 & 5251.27 & 5.329 & 4.319 & 3.662 & 2.667 & 8086.58 & 6.295 & 5.008 & 4.042 & 2.674 \\
\hline 2881.50 & 6.73 & 5.60 & 4.74 & 3.37 & 5481.63 & 6.238 & 5.196 & 4.420 & 3.344 & 8115.49 & 6.526 & 5.230 & 4.254 & 2.907 \\
\hline 2912.50 & 6.35 & 5.34 & 4.48 & 3.29 & 5543.43 & 6.223 & 5.180 & 4.404 & 3.274 & 8142.42 & 6.720 & 5.440 & 4.445 & 3.052 \\
\hline 2971.50 & 5.38 & 4.64 & 4.00 & 3.05 & 5546.45 & 6.173 & 5.133 & 4.365 & 3.259 & 8389.68 & 5.943 & 4.705 & 3.793 & 2.464 \\
\hline 2995.50 & 5.43 & 4.47 & 3.84 & 2.94 & 5565.38 & 6.155 & 5.107 & 4.336 & 3.247 & 8452.45 & 6.060 & 4.757 & 3.852 & 2.588 \\
\hline 3012.50 & 5.40 & 4.42 & 3.82 & 2.90 & 5570.36 & 6.090 & 5.080 & 4.314 & 3.206 & 8500.43 & 6.527 & 5.172 & 4.201 & 2.846 \\
\hline 3051.50 & 5.46 & 4.49 & 3.88 & 3.03 & 5595.34 & 5.845 & 4.898 & 4.179 & 3.059 & 8787.59 & 7.166 & 5.622 & 4.338 & 2.716 \\
\hline 3062.50 & 5.55 & 4.57 & 3.96 & 3.10 & 5614.32 & 5.649 & 4.677 & 3.979 & 2.904 & 8817.53 & 6.939 & 5.443 & 4.262 & 2.723 \\
\hline 3083.50 & 5.66 & 4.65 & 4.08 & 3.18 & 5627.31 & 5.593 & 4.579 & 3.897 & 2.830 & 8854.40 & 6.819 & 5.398 & 4.298 & 2.806 \\
\hline 3284.68 & 6.09 & 5.18 & 4.40 & 3.31 & 5638.29 & 5.443 & 4.448 & 3.803 & 2.818 & 8875.39 & 6.876 & 5.469 & 4.391 & 2.905 \\
\hline 3322.55 & 5.62 & 4.74 & 4.09 & 3.09 & 5849.61 & 6.237 & 5.186 & 4.439 & 3.399 & 8932.30 & 7.255 & 5.921 & 4.828 & 3.257 \\
\hline 3346.45 & 5.46 & 4.53 & 3.88 & 2.99 & 5873.60 & 6.271 & 5.223 & 4.468 & 3.448 & 9146.69 & 6.420 & 5.195 & 4.199 & 2.753 \\
\hline 3386.38 & 5.33 & 4.37 & 3.77 & 2.97 & 5893.52 & 6.210 & 5.189 & 4.444 & 3.442 & 9171.50 & 6.182 & 4.969 & 4.036 & 2.656 \\
\hline 3412.32 & 5.34 & 4.36 & 3.78 & 2.91 & 5906.59 & 6.159 & 5.130 & 4.394 & 3.402 & 9215.47 & 5.922 & 4.731 & 3.866 & 2.599 \\
\hline 3431.31 & 5.45 & 4.45 & 3.85 & 3.05 & 5924.42 & 6.092 & 5.079 & 4.353 & 3.377 & 9586.50 & 5.675 & 4.471 & 3.659 & 2.514 \\
\hline 3439.32 & 5.41 & 4.44 & 3.87 & 3.04 & 5957.35 & 6.144 & 5.131 & 4.391 & 99.999 & 9640.33 & 5.681 & 4.480 & 3.702 & 2.588 \\
\hline 3644.64 & 6.49 & 5.56 & 4.68 & 3.42 & 6029.27 & 5.429 & 4.461 & 3.829 & 2.844 & 9890.70 & 6.125 & 4.959 & 4.022 & 2.675 \\
\hline 3655.64 & 6.41 & 5.48 & 4.60 & 3.38 & 6225.55 & 6.138 & 5.062 & 4.351 & 3.409 & 9937.46 & 5.725 & 4.528 & 3.658 & 2.442 \\
\hline 3662.67 & 6.40 & 5.44 & 4.59 & 3.46 & 6266.49 & 6.255 & 5.191 & 4.448 & 3.489 & 10006.39 & 5.460 & 4.289 & 3.505 & 2.392 \\
\hline 3675.65 & 6.20 & 5.32 & 4.50 & 3.35 & 6351.32 & 5.815 & 4.870 & 4.166 & 3.163 & 10016.27 & 5.481 & 4.313 & 3.542 & 2.433 \\
\hline 3695.58 & 5.93 & 5.03 & 4.27 & 3.17 & 6597.56 & 6.366 & 5.229 & 4.414 & 3.347 & 10212.66 & 6.738 & 5.486 & 4.458 & 3.067 \\
\hline 3706.55 & 5.78 & 4.90 & 4.17 & 3.06 & 6645.50 & 6.928 & 5.740 & 4.789 & 3.522 & 10256.62 & 6.725 & 5.458 & 4.397 & 3.004 \\
\hline 3729.43 & 5.67 & 4.73 & 4.02 & 2.98 & 6693.40 & 6.988 & 5.675 & 4.743 & 3.371 & 10299.50 & 6.482 & 5.102 & 4.004 & 2.629 \\
\hline 3819.26 & 5.42 & 4.41 & 3.83 & 2.89 & 6704.36 & 6.919 & 5.721 & 4.713 & 3.347 & 10590.65 & 7.951 & 6.371 & 4.976 & 3.278 \\
\hline 3821.26 & 5.49 & 4.48 & 3.88 & 2.89 & 6748.32 & 6.718 & 5.576 & 4.600 & 3.242 & 10673.35 & 7.290 & 5.678 & 4.348 & 2.780 \\
\hline 4070.53 & 5.61 & 4.70 & 4.05 & 3.06 & 6923.65 & 6.168 & 4.912 & 4.051 & 2.872 & 10995.51 & 7.864 & 6.498 & 5.173 & 3.392 \\
\hline 4096.48 & 5.27 & 4.37 & 3.77 & 2.85 & 6959.58 & 6.660 & 5.346 & 4.404 & 3.140 & 11057.42 & 7.516 & 6.060 & 4.695 & 2.946 \\
\hline 4364.62 & 6.35 & 5.30 & 4.51 & 3.43 & 6985.51 & 7.064 & 5.726 & 4.727 & 3.359 & 11104.55 & 7.174 & 5.718 & 4.430 & 2.783 \\
\hline 4429.57 & 6.16 & 5.16 & 4.37 & 3.29 & 6999.52 & 7.293 & 5.940 & 4.906 & 3.442 & 11389.45 & 7.694 & 6.322 & 5.106 & 3.395 \\
\hline 4434.56 & 6.16 & 5.16 & 4.36 & 3.26 & 7025.39 & 7.688 & 6.333 & 5.182 & 3.596 & 11417.44 & 7.621 & 6.246 & 5.012 & 3.285 \\
\hline 4445.48 & 6.05 & 5.05 & 4.28 & 3.21 & 7072.37 & 8.093 & 6.657 & 5.307 & 3.573 & 11685.60 & 7.867 & 6.468 & 5.234 & 3.534 \\
\hline 4744.65 & 6.247 & 5.254 & 4.423 & 3.323 & 7331.55 & 7.435 & 5.905 & 4.623 & 3.014 & 11712.62 & 7.919 & 6.518 & 5.267 & 3.569 \\
\hline 4771.62 & 6.232 & 5.222 & 4.357 & 3.228 & 7357.45 & 7.762 & 6.207 & 4.892 & 3.240 & 11743.53 & 7.760 & 6.373 & 5.149 & 3.457 \\
\hline 4772.53 & 6.192 & 5.194 & 4.321 & 3.156 & 7379.50 & 8.001 & 6.491 & 5.126 & 3.394 & 11783.42 & 7.597 & 6.232 & 5.018 & 3.320 \\
\hline 4777.56 & 6.174 & 5.187 & 4.319 & 3.173 & 7393.38 & 8.200 & 6.688 & 5.323 & 3.532 & 11814.38 & 6.898 & 5.646 & 4.542 & 2.944 \\
\hline 4781.54 & 6.178 & 5.204 & 4.333 & 3.196 & 7394.36 & 8.229 & 6.687 & 5.293 & 3.528 & 11828.33 & 6.674 & 5.419 & 4.364 & 2.835 \\
\hline 4803.54 & 6.173 & 5.160 & 4.267 & 3.135 & 7438.25 & 8.582 & 7.041 & 5.554 & 3.634 & 11860.24 & 6.408 & 5.146 & 4.141 & 2.731 \\
\hline 4815.45 & 6.141 & 5.159 & 4.265 & 3.095 & 7657.63 & 7.284 & 5.699 & 4.364 & 2.702 & 12066.63 & 7.783 & 6.409 & 5.257 & 3.614 \\
\hline 4822.49 & 6.071 & 5.073 & 4.201 & 3.096 & 7690.61 & 7.411 & 5.816 & 4.506 & 2.854 & 12087.59 & 7.945 & 6.497 & 5.286 & 3.632 \\
\hline 4899.31 & 5.113 & 4.199 & 3.534 & 2.591 & 7719.55 & 7.537 & 5.978 & 4.681 & 3.006 & 12117.52 & 7.970 & 6.514 & 5.252 & 3.571 \\
\hline 5100.66 & 6.185 & 5.119 & 4.345 & 3.366 & 7744.52 & 7.709 & 6.160 & 4.876 & 3.177 & 12155.44 & 7.868 & 6.423 & 5.107 & 3.358 \\
\hline 5157.52 & 5.933 & 4.932 & 4.181 & 3.148 & 7776.39 & 7.916 & 6.392 & 5.119 & 3.417 & 12183.38 & 7.555 & 6.135 & 4.871 & 3.197 \\
\hline 5193.43 & 5.882 & 4.911 & 4.168 & 3.048 & 7811.35 & 8.004 & 6.490 & 5.259 & 3.558 & 12208.25 & 7.143 & 5.744 & 4.543 & 2.912 \\
\hline
\end{tabular}

\section{Results and discussion}

\subsection{Comparison of visual and IR data}

In all four smoothed IR light curves in Fig. 2, what we call the events I, II and III, can clearly be resolved as outstanding features, indicating that during these periods the Mira was heavily obscured by dust. The amplitudes of the variations are lower at the longer wavelengths. In addition, there are clear differences between the $L$ and the other IR light curves, the $L$ mag being approximately constant with only very slight obscuration features.

There is no need to correct the AAVSO curve for Mira pulsations which are not seen in the optical region, because the unobscured parts of the nebula are the dominant source of emission at these short wavelengths. Let us recall that the nebulae within the system have a very complex structure (Nussbaumer 2000). In the part of the AAVSO curve shown in Fig. 1 there are three separate segments in time intervals $\mathrm{a}, \mathrm{b}$ and $\mathrm{c}$. The fadings over intervals a and $\mathrm{c}$ are at approximately the same rate, in contrast to the period $b$ during which the visual magnitude remained almost constant. In general, the AAVSO curve shows very little relation to the IR variations. The dust obscuration event starting about JD 2450000 may be connected with the fading visual luminosity, the effect being larger in $J$ than in other near-IR bands.
The changes in the IR and visual light curves are summarized in the Table 2. The depth of the obscuration in $J H K$ and $L$ is measured with respect to the pre-obscuration level during 1983 through to 1986 with the assumption that it reasonably represents the mean brightness of the Mira. Clearly the assumption is better for the first obscuration phases than for the later ones.

\subsection{JHKL colours}

Figure 3 shows the evolution of the $J-H, J-K$ and $J-L$ colour indices, which are strongly influenced by the presence of dust around the system. Their maxima clearly indicate the epochs of maximum obscuration.

More detailed information on the colour behaviour of RR Tel, concerning differences between the three obscuration phases and the epochs preceding them, can be derived from Fig. 4. The best statistical fit to the $J, H, K, L$ correlations indicates no difference among the correlations during the three obscuration events in each band. The correlation gradients have the same sign in all bands, being smaller when the wavelength with which $J$ is correlated is larger. This is to be expected in the presence of absorption due to dust, where the absorption will decrease with wavelength. The correlations suggest a change 
Table 2. The features and time correlation of the IR and visual light curves.

\begin{tabular}{|c|c|c|c|c|c|c|c|c|c|}
\hline \multicolumn{6}{|c|}{ IR photometry } & \multicolumn{2}{|c|}{ Time } & \multicolumn{2}{|c|}{ Visual photometry } \\
\hline \multirow[t]{2}{*}{ epoch } & \multicolumn{4}{|c|}{$\Delta m$} & \multirow[t]{2}{*}{ phase } & \multicolumn{2}{|c|}{ JD-2 440 000 date } & \multirow{2}{*}{ epoch } & \multirow[t]{2}{*}{$m$} \\
\hline & $J$ & $H$ & $K$ & $L$ & & & & & \\
\hline & & & & & & 4150 & Oct. 1979 & $\mathrm{a}$ & 10,00 \\
\hline & & & & & & 5300 & Nov. 1984 & & 10,30 \\
\hline \multirow{3}{*}{ I } & \multirow{3}{*}{2,6} & \multirow{3}{*}{2,0} & \multirow{3}{*}{1,1} & \multirow{3}{*}{0,2} & start & 6600 & Jun. 1986 & & 10,60 \\
\hline & & & & & & 7000 & Aug. 1987 & & 10,75 \\
\hline & & & & & minimum & 7700 & Jun. 1989 & \multirow{3}{*}{$\mathrm{b}$} & 10,75 \\
\hline \multirow{3}{*}{ II } & \multirow{3}{*}{2,2} & \multirow{3}{*}{1,5} & \multirow{3}{*}{0,8} & \multirow{3}{*}{0,0} & start & 8400 & May 1991 & & 10,75 \\
\hline & & & & & minimum & 8800 & Jun. 1992 & & 10,75 \\
\hline & & & & & & 10000 & Nov. 1995 & & 10,75 \\
\hline \multirow{3}{*}{ III } & \multirow{3}{*}{2,2} & \multirow{3}{*}{1,7} & \multirow{3}{*}{1,0} & \multirow{3}{*}{0,2} & start & 10200 & May 1996 & \multirow{3}{*}{$\mathrm{c}$} & 10,75 \\
\hline & & & & & minimum & 11100 & Oct. 1998 & & 11,05 \\
\hline & & & & & & 12440 & Jun. 2002 & & 11,50 \\
\hline
\end{tabular}

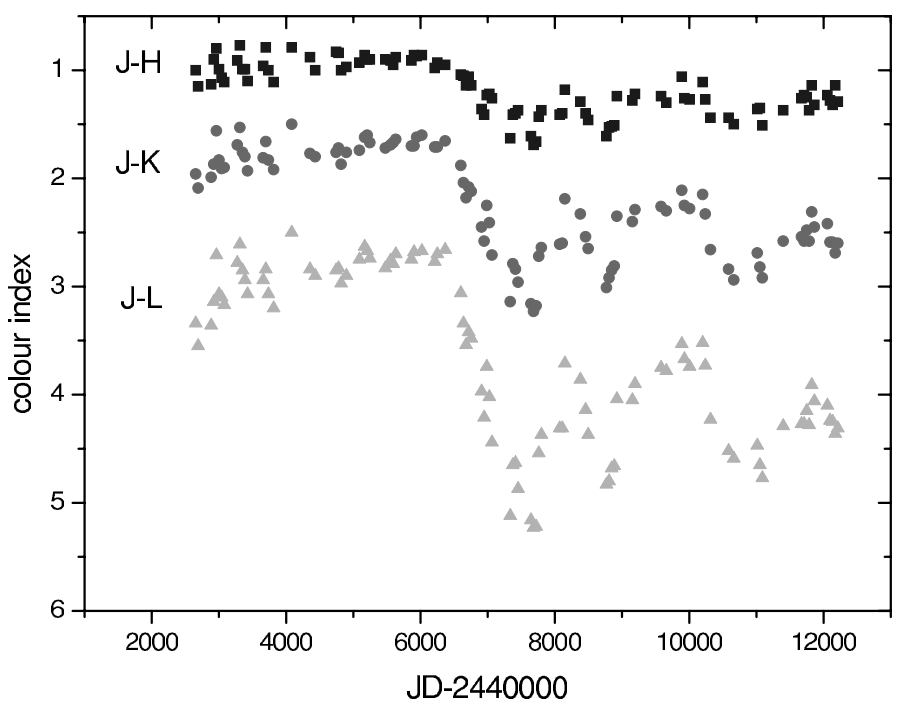

Fig. 3. Time evolution of the $J-H, J-K$ and $J-L$ colour indices.

in colour behaviour around JD 2446600 , the colours becoming redder in the later period. The change, which was not sudden is clearest in the $L$ band where RR Tel is brightest. This could result from grain fragmentation and grain vaporization which will create an excess of small dust particles in a low velocity $\left(V<200 \mathrm{~km} \mathrm{~s}^{-1}\right)$ shock regime compared to the unshocked one. However, we would need to have infrared spectra for the same epochs to be sure that no other effect is involved.

It is instructive to compare the colours of RR Tel with the mean values of normal Miras. The RR Tel $J-H, H-K$ and $J-K, K-L$ two colour diagrams as well as those for Miras with mainly thin dust shells (Whitelock et al. 2000) and for IRAS selected Miras with relatively thick dust shells (Whitelock et al. 1994) are shown in Figs. 5 and 6. It is clear that the observed RR Tel infrared colours are significantly shifted to the right of the range shown by normal Miras.

The colours of RR Tel can be explained by reddening which dominates over a small amount of dust emission. This interpretation was discussed by Whitelock (1987), who presented a sample of symbiotic binaries in a two-colour diagram, modeling their colours with a $2500 \mathrm{~K}$ star plus an $800 \mathrm{~K}$ dust shell (represented as a locus on her Fig. 3). She discussed the mean colours of six symbiotics in and out of obscuration events and noted that they tended to move higher up the locus during the events. This is

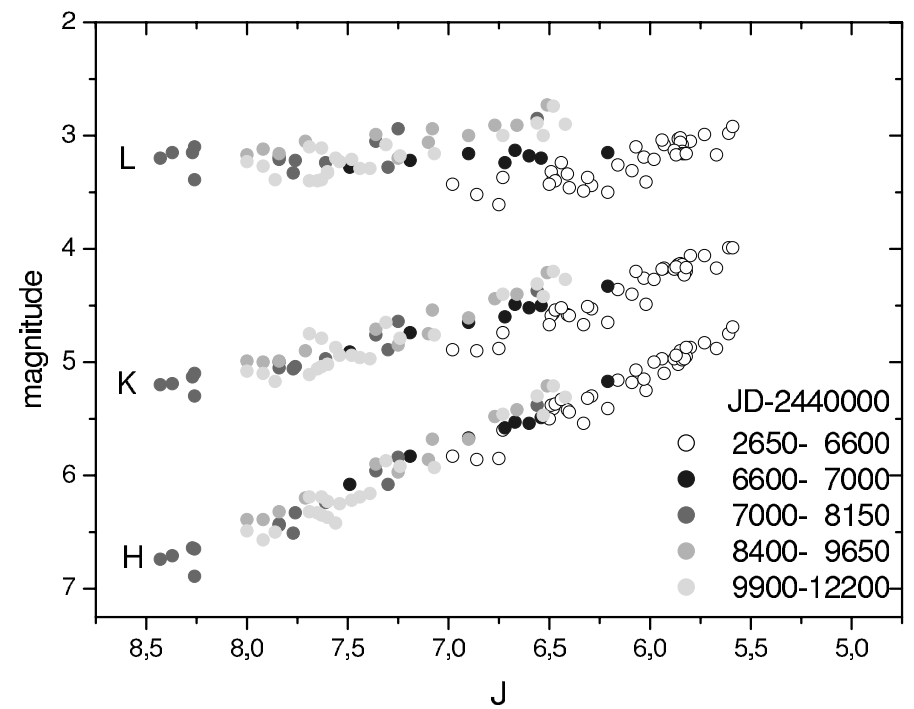

Fig. 4. Correlation of the $H, K$ and $L$ magnitudes with $J$ for different time periods. Empty symbols refer to dates before JD 2446600 . The transition epoch (JD 2446 600-2 447 000) characterized by the gradual change in the behaviour of the colour variations and the following three obscuration events I, II and III are distinguished by different grey tones.

consistent with the obscuration being caused by increased dust absorption in the line of sight. The locus, represented in our twocolour $J-K, K-L$ diagram, was calculated on the assumption that silicate dust surrounds the Mira whose radiation it absorbs and re-emits at the given temperature. The further up the locus, the thicker is the dust shell. RR Tel follows the same type of trend as the other objects showing obscuration events.

The changes in the colours of RR Tel are consistent with their being caused by changes in the optical depth of a silicate dust shell (with grain properties from Le Bertre et al. 1984, Table 2) which re-radiates predominantly around $700 \mathrm{~K}$ (see Fig. 6).

This is of course very much an oversimplification, but it produces a good first-order fit to the near-infrared colours. The displacement of RR Tel, like that of other symbiotic Miras, with respect to the single Miras, is according to the present model, a consequence of the higher temperature (600-800 K) of the dust shell around the cool component (Danchi et al. 1994), compared with temperatures $100-300 \mathrm{~K}$ of non-symbiotic Miras (Whitelock 1987). 


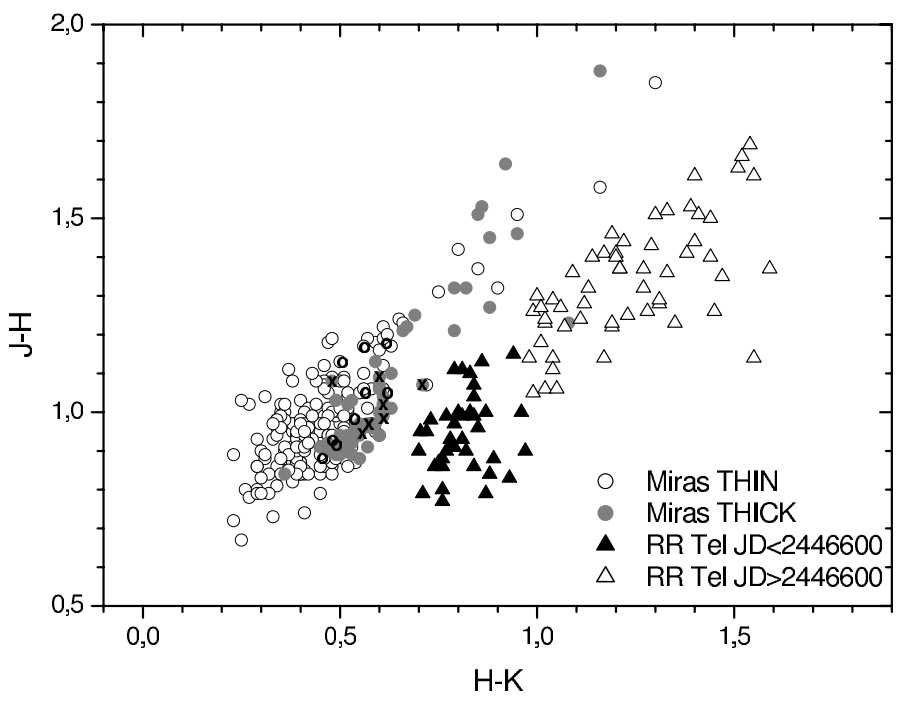

Fig. 5. Two colour $J-H, H-K$ diagram of RR Tel before (full triangles) and after (empty triangles) JD 2446600 , as well as of normal Miras with thin dust shells (empty circles) from Whitelock et al. (2000) and with thick dust shells (full circles) from Whitelock et al. (1994). The Miras with similar periods of pulsation to that of RR Tel are distinguished by crosses for thick dust shells and by bold circles for thin dust shells. Straight lines are least square fits for RR Tel before and after JD 2446600 .

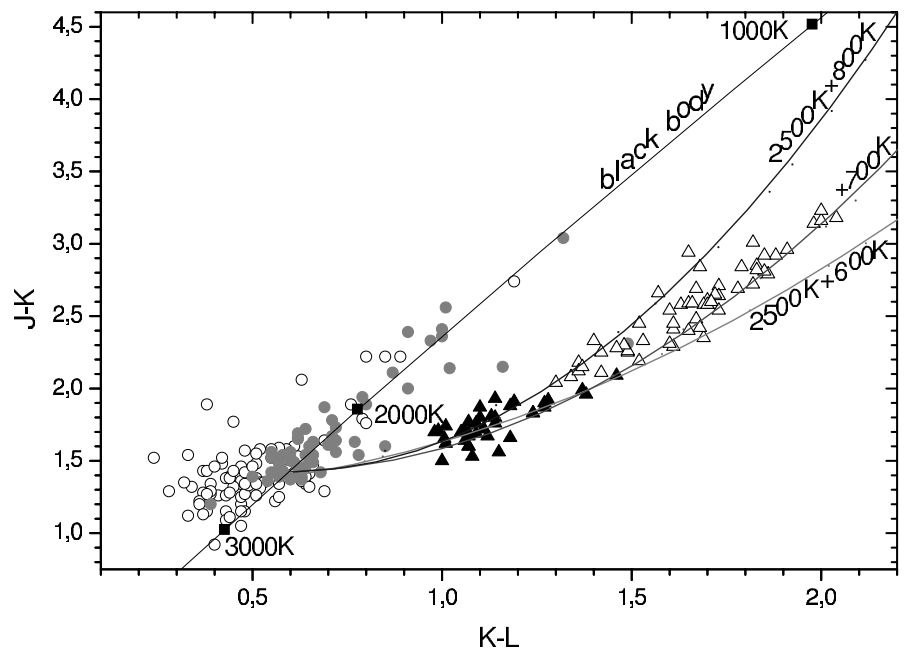

Fig. 6. Two colour $J-K, K-L$ diagram of RR Tel. In addition to the same symbols as in Fig. 5, the black body curve and and curves representing a $2500 \mathrm{~K}$ Mira $+800 \mathrm{~K}, 700 \mathrm{~K}$ and $600 \mathrm{~K}$ silicate dust shell of variable optical depth are plotted.

We wonder if the extra heating is provided by the hot component of the binary system as long as its temperature is not too high to evaporate the dust grains. Actually, the theoretical models of Danchi et al. (1994) indicate relatively high dust temperatures $(>500 \mathrm{~K})$ also in dust shells around isolated Mirae.

In Table 3 we compare the extinction in obscuration phase I with that expected from interstellar extinction according to the Rieke \& Lebofsky law (Rieke \& Lebofsky 1985) and the van der Hulst curve 15 (vdH15). We also show for comparison the extinction experienced by the symbiotic Mira R Aqr (Whitelock et al. 1983) during its 1976 obscuration event. All values have been normalized to $\Delta J=1$.

A comparison of the figures in Table 3 suggests that the obscuration at $J H K$ is plausibly caused by extinction by particles

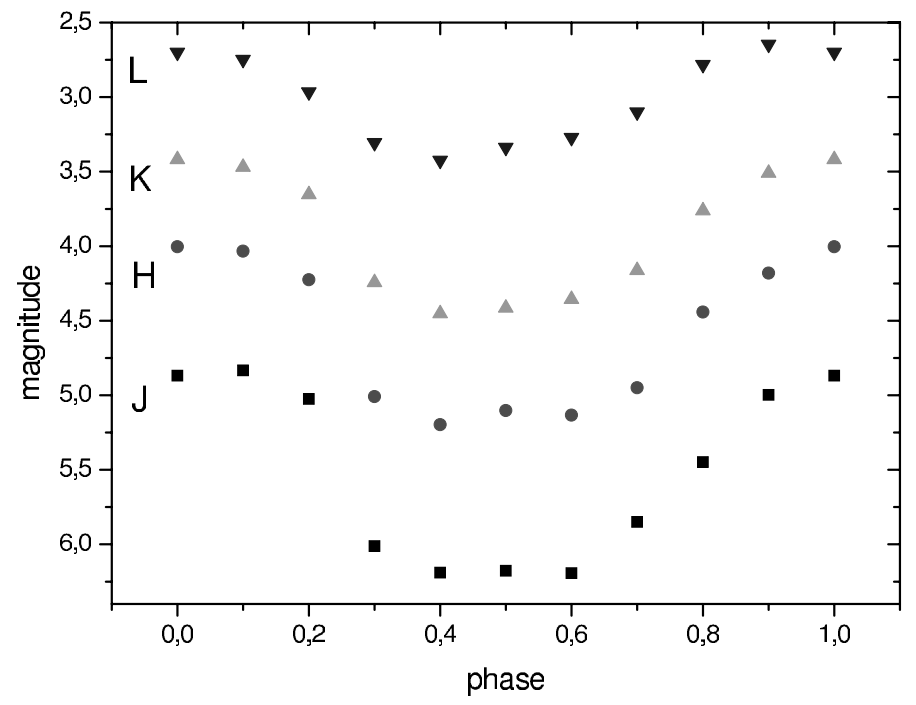

Fig. 7. Mira pulsations of RR Tel at different wavelengths.

Table 3. Wavelength dependence of extinction during obscuration phases.

\begin{tabular}{ccccc}
\hline \hline$\Delta J$ & $\Delta H$ & $\Delta K$ & $\Delta L$ & Source \\
\hline 1.0 & 0.77 & 0.42 & 0.08 & RR Tel phase I (this work) \\
1.0 & 0.63 & 0.36 & 0.18 & vdH 15 (Rieke \& Lebofsky 1985) \\
1.0 & 0.80 & 0.51 & 0.26 & Rieke \& Lebofsky (1985) \\
1.0 & 0.8 & 0.6 & 0.3 & R Aqr 1976 (Whitelock et al. 1983) \\
\hline
\end{tabular}

comparable to those found in the interstellar medium. However, the fact that the extinction at $L$ is rather less than predicted by normal reddening laws and is unlike the case of $\mathrm{R}$ Aqr, may suggest somewhat different grain sizes.

Comparison of the amplitudes of variation (Fig. 7) with the mean colours shows no clear difference between the Mira component of RR Tel and single Miras. Particularly RR Tel fits well into the correlation between the pulsation amplitude in the $K$ band and $K-L^{\prime}$ of Le Bertre (1993), but shows a somewhat larger $K$ pulsation amplitude relative to all the oxygen-rich latetype stars of the sample in the period vs. $K$ pulsation amplitude correlation. Olivier et al. (2001) also studied the amplitudes of variation of dust enshrouded AGB stars. The amplitude of the Mira component of RR Tel is larger than that expected for stars of about the same pulsation period according to their periodamplitude correlation, except for one C-rich object which shows about the same deviation in the $L$ band. The Mira pulsation amplitudes in the two correlations between amplitudes in different bands appear to be fairly normal according to the values of Smith (2003).

The contribution of other non-Mira sources of radiation to the infrared magnitudes and especially to $J$, is not easy to see. One way to study it, would in principle be to compare the apparent amplitude of the Mira pulsations during obscuration events to that outside obscuration events. The proportion of radiation coming from the Mira would be less at Mira minimum during an obscuration event than outside it, so decreasing the apparent Mira amplitude. Figure 8 shows the Mira $J$ light curve, which appears to be similar at different times. However, the method is in our case not reliable enough because the Mira light-curve interpolation procedure does not work very well during obscuration events. Nevertheless, we doubt that the hot component is responsible for the systematic effects in the near IR. One reason 


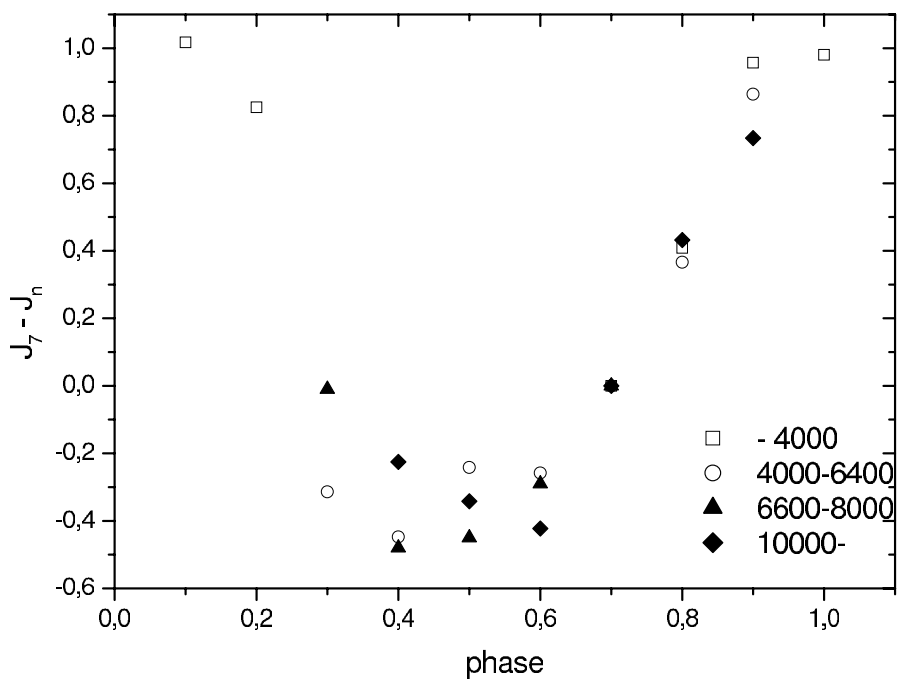

Fig. 8. The Mira pulsations in $J$ at different epochs before the start of obscuration event I at JD 2446600 (empty symbols) and after it (full symbols): till JD 2444000 (squares), JD 2444 000-JD 2446400 (circles), JD $2446600-J D 2448000$ (triangles), after JD 2450000 (diamonds). The magnitudes at each phase $J_{7}-J_{n}$ are given relative to that at phase 0.7 .

is the lack of evidence of a strong continuum in the optical and UV (Penston et al. 1983).

The nebular component will contribute mainly through the emission lines and through the bremsstrahlung which dominates in the radio range.

The infrared spectrum of RR Tel (Feast et al. 1983) shows strong water absorption without obvious emission lines and so suggests that at a Mira phase of 0.8 (Belczynski et al. 2000) the radiation from the cool component is the major contributor in $J$.

Other evidence for a negligible contribution from anything other than the Mira to the $J$ flux is the large amplitude of the dust obscuration events seen in Fig. 2. The largest $J$ amplitude is 2.6 mag (factor of 11), with other events of smaller amplitude. On the other hand, the strongest absorption feature due to water vapour presumably due to the Mira, seen in the rather low resolution infrared spectrum of Feast et al. (1983) at $1.4 \mu \mathrm{m}$, has an apparent depth of about 60 percent of the continuum. That spectrum of 1980 Aug. 20 was taken when dust obscuration was near minimum. The disappearance of all the flux below the absorption feature, even if one supposes it only due to the nebular component, would limit the effect of dust obscuration to $0.55 \mathrm{mag}$, assuming that obscuration does not affect this nebular component.

\subsection{Optical Fe II emission lines}

The fluxes of the permitted Fe II and forbidden [Fe II] lines, in the spectra taken in 1996 and 2000, were measured and compared. The spectra taken in 2000 were corrected for reddening using the reddening law of Howarth (1983) with $R=3.1$ and $E(B-V)=0.08$. Crawford corrected the 1996 spectra for interstellar extinction using the coefficients listed in Cardelli et al. (1989) and $E(B-V)=0.08$.

The dates of the spectra are marked in the corresponding segment of the $J$ and visual light curves which are plotted in Figs. 9 and 10 respectively. A dust obscuration event was just starting when the 1996 spectra were taken and underway when the 2000 spectra were obtained. Correcting for the Mira

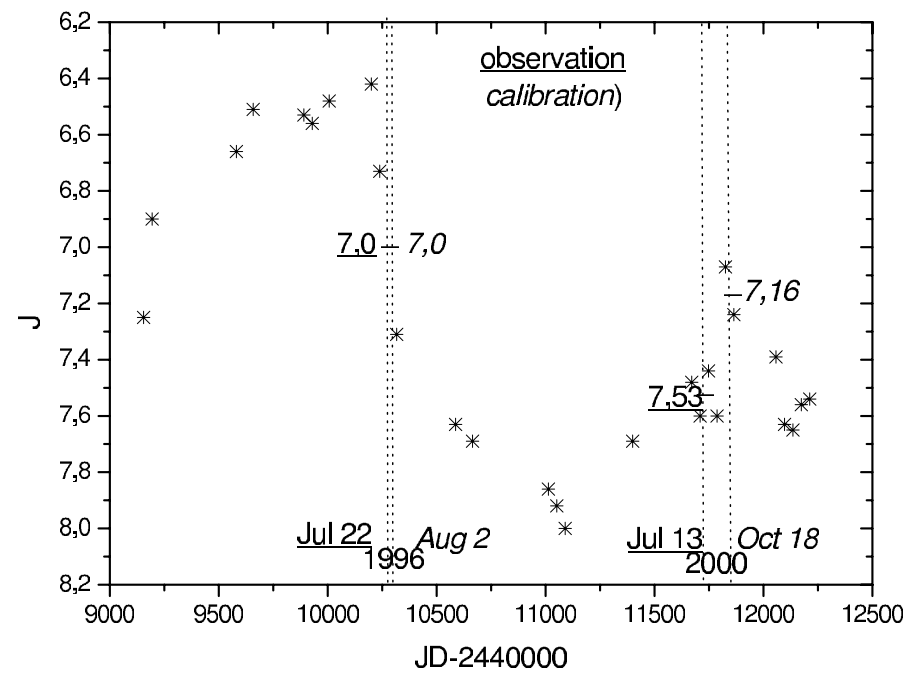

Fig. 9. $J$ light curve corrected for Mira pulsations during the dust obscuration episode when the two sets of optical spectra, 1996 and 2000, were taken.

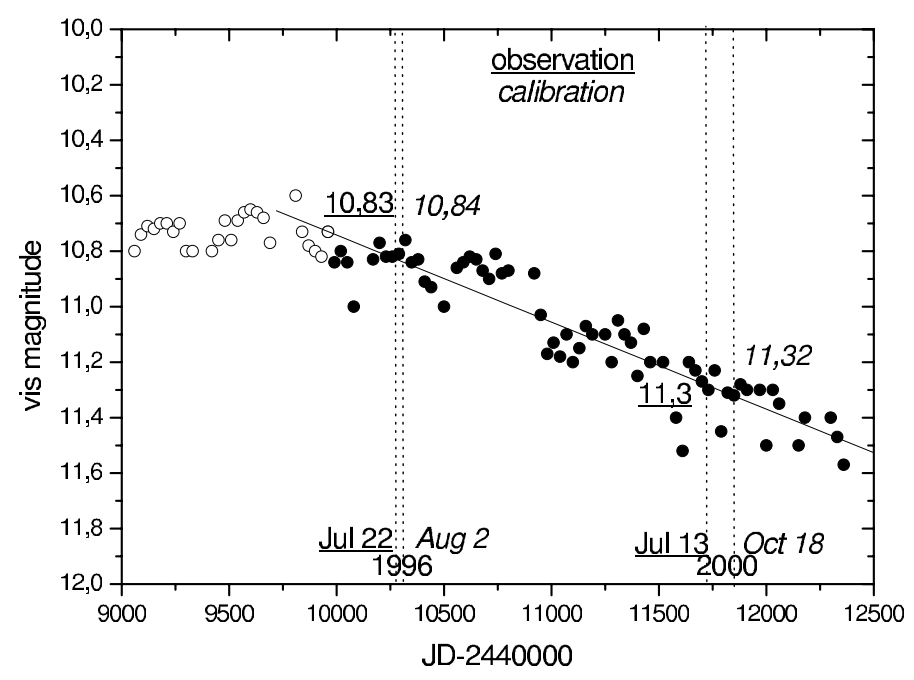

Fig. 10. Visual light curve of RR Tel during the dust obscuration episode when the two optical spectra, 1996 and 2000, were taken.

pulsations, a fading of only 0.16 mag in $J$ is obtained between the times of the calibration spectra, because of a temporary $J$ brightening when the calibration of the later spectrum was obtained (Fig. 9). The observed visual fading between the same two dates was 0.48 mag (Fig. 10).

It might be interesting to compare the fading in $V$ due to many contributions, depending on changing physical conditions as well as on the obscuration event, with the Fe II line and infrared fadings.

Other lines from different levels (Kotnik-Karuza et al. 2004a,b) behave differently because they are sensitive in different ways to the physical conditions of the emitting gas.

According to an interstellar extinction law (Rieke \& Lebofsky 1985), the fading of 0.16 in $J$ would correspond to a fading of 0.57 mag in $V$.

The latter corresponds to a flux decrease given by a log flux ratio equal to -0.23 in the $V$ band if the radiation in $J$ and $V$ came from the same region and was absorbed by the same medium. Log flux ratios between the two dates for the Fe II and [Fe II] emission line fluxes in the two spectra, plotted against 


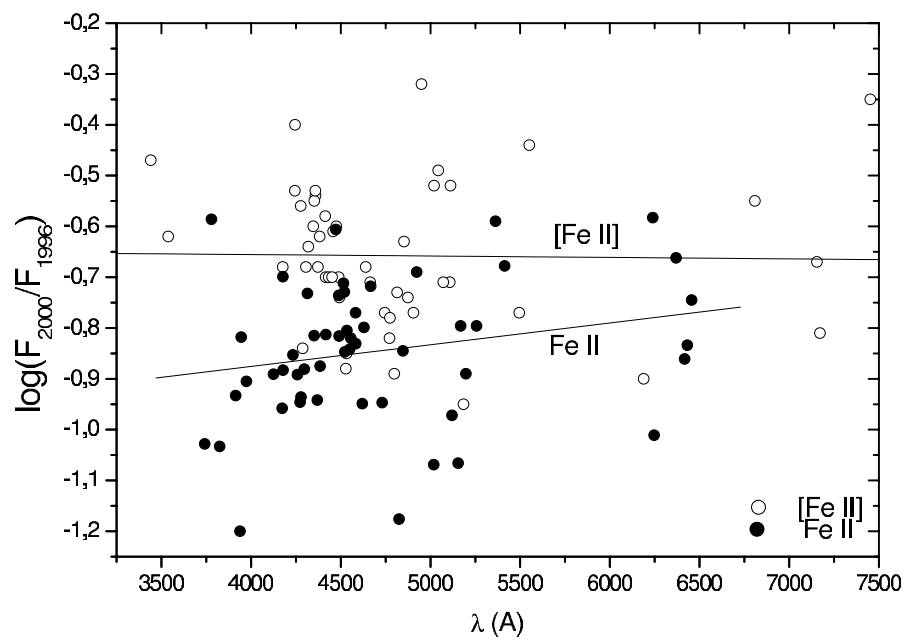

Fig. 11. Fading of Fe II and [Fe II] log line fluxes from 1996 to 2000 as a function of wavelength.

wavelength, are shown in Fig. 11. Lines with wavelengths below $3490 \AA$ were not taken into account in our iron line study, as the Crawford calibration in that region is highly uncertain. The measured line of multiplet 73 at a large wavelength of $7711 \AA$ and a few other weak extremely discordant lines were also eliminated. Only the difference between the permitted and forbidden lines appears to be significant, showing a larger flux decrease for the permitted than for the forbidden lines. No significant correlation has been found between other line properties and log ratio. The best correlation between the log flux ratio of the forbidden lines and $\log (g f \lambda)$ still has a probability of 0.25 and would moreover be difficult to understand for optically thin forbidden lines. The mean $\log$ flux ratios are $-0.85 \pm 0.14$ for Fe II and $-0.66 \pm 0.14$ for [Fe II].

Note that the log flux change of the iron lines between the two spectra is much larger than the change in the $V$ band over the same period. It therefore seems possible that these lines are formed closer to the Mira than the higher temperature region responsible for most of the emission at $V$.

Let us note that the narrowness of the Fe II and [Fe II] emission lines, found by Kotnik-Karuza et al. (2002), indicates line formation in a low velocity wind of the cool component.

Looking for the simplest possible interpretation of what we see and especially Fig. 11, two types of model might be considered. One involves spherical symmetry of the extra dust absorption in the Mira wind, while the other assumes the presence of one or more clouds. In both cases the apparent radius of the line emitting region found by the Self-Absorption Curve (SAC) method, might be expected to decrease during a dust obscuration episode (Kotnik-Karuza et al. 2002, 2003). In both cases the presumably less absorbed forbidden line region will be larger.

The log optical absorption of 0.23 , corresponding to the $J$ absorption, is less than that of the Fe II lines. One might think that this disagreement is due to the difficulty of finding the exact $J$ magnitudes when the spectra were taken, so the spherically symmetric model, with fewer grains above the forbidden line region than the permitted line one, is not then necessarily contradicted.

We must, nevertheless, emphasize that the low velocity of the Mira wind should lead to the line formation regions being occulted by dust significantly later than the Mira itself, so direct comparison of the $J$ and line flux fadings may be misleading.
The lack of a clear wavelength dependence of the extra absorption of the optical lines, unlike the behaviour shown in the near infra-red, is, however, a problem and might suggest rather the presence of one or more optically thick clouds occulting much of the Fe II line emitting regions.

\subsection{Distance estimates}

Studies of the optical emission spectrum of RR Tel suggest considerably more dust toward the cool star than on the line of sight to the high excitation regions (Kotnik-Karuza et al. 2004b). Thus, in determining the distance to the star, one should take into account the fact that the extinction in the direction of the cool component includes circumstellar as well as interstellar extinction. The interstellar extinction towards RR Tel is $E(B-V)=0.10-0.11$ (Penston et al. 1983; Young et al. 2005) which would give $A_{K} \sim 0.03 \mathrm{mag}$.

For the distance estimate we use the mean values of the $K$ magnitude and of the $J-K$ colour index equal to 4.16 and 1.69 , respectively, obtained from observations at the epoch preceding the first obscuration event (I), when the circumstellar absorption was at its minimum.

From the value of 387 days for the period of Mira pulsations and also using the correlation between the Mira period and absolute magnitude (Feast et al. 1989, assuming a distance modulus for the LMC of $18.5 \mathrm{mag}$ ), we obtain an absolute magnitude in $K, M_{K}=-8.0$. The unabsorbed $J-K$ was obtained by applying the period-colour relation for Mira variables in the solar neighbourhood from Whitelock et al. (2000), $(J-K)_{0}=-0.39+$ $0.71 \log P$. This leads to $(J-K)_{0}=1.45$ and $E_{J-K}=0.24$. Using the reddening law specified by Glass (1999) we find $A_{K}=0.13$ - indicating that most of the obscuration is circumstellar rather than interstellar.

Thus the distance to RR Tel is $2.5 \mathrm{kpc}$, which is very close to the value of $2.6 \mathrm{kpc}$ found e.g. by Whitelock (1988). This procedure assumes that the circumstellar extinction has the same reddening characteristics as does interstellar extinction. In view of the discussion of the extinction during the obscuration phases (Sect. 3.2) and the fact that the correction is small, the assumption is unlikely to cause serious errors in the distance, which should be good to about $0.3 \mathrm{kpc}$.

\section{Conclusions}

Our study, of both broad band fluxes and the fluxes of emission lines of once ionized iron, lead to suggestive results, whose interpretation is, however, still uncertain. The infrared flux variations are understandable in terms of wavelength dependent absorption by dust around the cool component. It is not clear what the reason is for the change in behaviour of fluxes between JD 2446600 and JD 2447000 . The effect with respect to $J$ is larger for longer wavelength bands, suggesting that the continuum absorption of dust is responsible, rather than changes of emission line fluxes.

The two-colour diagrams show unusual characteristics for RR Tel compared with the colours of single Miras. The track during the fading events is consistent with obscuration caused by an increasingly thick dust shell. The characteristic temperature of the dust shell, $700 \mathrm{~K}$, is sufficiently hot to produce an excess in the $L$ band which results in the observed colours. A qualitatively similar explanation was offered for the colours of symbiotic Miras by Whitelock (1987). 
The relatively high temperature found for the dust shell could be a consequence of heating by the binary companion. A consistent model is needed to check this hypothesis.

The optical magnitudes are not simply correlated with the infrared ones, but later fading might be connected with dust obscuration. It should be noted that the optical behaviour is governed by the behaviour of the different emission line fluxes.

The apparently wavelength independent variation of the fluxes of the lines of ionized iron during dust obscuration is most simply understood as being due to absorption by separate optically thick clouds.

If the narrow ionized iron lines (Kotnik-Karuza et al. 2003) are formed in the cool star's wind, the lines could be formed farther out from the cool component than the dust producing the infrared broad band absorption. If fewer clouds absorb, the then less absorbed forbidden lines appear to be, as expected, formed farther out than the permitted lines. However, we do not have enough observations at different epochs; ionized iron and perhaps other emission line fluxes should rise at the end of obscuration events, if the present interpretation is correct. We should note that the presence of separate clouds is not unexpected, as it is rather similar to what is thought to occur during fadings of $\mathrm{R}$ Corona Borealis stars (Feast 1986).

Acknowledgements. We are grateful to Michael Feast for starting the IR monitoring programme at SAAO. Our thanks are also due to the late Janet Mattei, Rebecca Pollock and to Albert Jones for providing us with the optical photometry of RR Tel.

\section{References}

Belczynski, K., Mikolajewska, J., Munari, U., Ivison, R. J., \& Friedjung, M. 2000, A\&AS, 146, 407

Cardelli, J. A., Clayton, G. C., \& Mathis John, S. 1989, ApJ, 345, 245

Carter, B. S. 1990, MNRAS, 242, 1

Contini, M., \& Formiggini, L. 1999, ApJ, 517, 925

Crawford, F. L., McKenna, F. C., Keenan, F. P., et al. 1999, A\&AS, 139, 135

Danchi, W. C., Bester, M., Degiacomi, C. G., Greenhill, L. J., \& Townes, C. H. 1994, AJ, 107 (4), 1469

Feast, M. W. 1986, in Hydrogen Deficient Stars and Related Objects, ed. K. Hunger, et al. (D. Reidel Publishing Company), 151
Feast, M. W., \& Whitelock, P. A. 1999, in Post-Hipparchos Cosmic Candles, ed. A. Heck, \& F. Caputo (The Netherlands: Kluwer Academic Publisher), 75

Feast, M. W., Robertson, B. S. C., \& Catchpole, R. M. 1977, MNRAS, 179, 499 Feast, M. W., Whitelock, P. A., Catchpole, R. M., Roberts, G., \& Carter, B. S. 1983, MNRAS, 202, 951

Feast, M. W., Glass, I. S., Whitelock, P. A., \& Catchpole, R. M. 1989, MNRAS, 241,375

Glass, I. S. 1999, Handbook of infrared astronomy, CUP

Heck, A., \& Manfroid, J. 1985, A\&A, 142, 341

Howarth, I. D. 1983, MNRAS, 203, 301

Kotnik-Karuza, D., Friedjung, M., \& Selvelli, P. L. 2002, A\&A, 381, 507

Kotnik-Karuza, D., Friedjung, M., Exter, K., Keenan, F. P., \& Pollacco, D. L. 2003, in Symbiotic stars probing stellar evolution, ed. R. L. M. Corradi, J. Mikolajewska, \& T. J. Mahoney, ASP Conf. Ser., 303, 136

Kotnik-Karuza, D., Friedjung, M., Exter, K., Keenan, F. P., \& Pollacco, D. L. 2004a, in Stars as Suns: Activity, Evolution, and Planets, ed. A. K. Dupree, \& A. Benz (San Francisco: Astronomical Sciety of the Pacific), CD-ROM, IAU Symp., 219, 888

Kotnik-Karuza, D., Friedjung, M., Exter, K., et al. 2004b, in Spectroscopically and Spatially Resolving the Components of Close Binary Stars, ed. R. W. Hidlitch, H. Hensberge, \& K. Pavlovski, ASP Conf. Ser., 318, 363

Le Bertre, T. 1993, A\&AS, 97, 729

Le Bertre, T., Epchtein, N., Gispert, R., \& Nguyen-Q-Rieu, Truong-Bach 1984, A\&A, 132, 75

McKenna, F. C., Keenan, F. P., Hambly, N. C., et al. 1997, ApJS, 109, 225

Mikolajewska, J., Brandi, E., Hack, W., et al. 1999, MNRAS, 305, 190

Mürset, U., \& Nussbaumer, H. 1994, A\&A, 282, 586

Mürset, U., Nussbumer, H., Schmid, H. M., \& Vogel, M. 1991, A\&A, 248, 458

Nussbaumer, H. 2000, in Thermal and Ionization Aspects of Flows from Hot Stars, ed. H. Lamers, \& A. Sapar, ASP Conf. Ser., 204, 317

Olivier, E. A., Whitelock, P., \& Marang, F. 2001, MNRAS, 326, 490

Penston, M. V., Benvenuti, P., Cassatella, A., et al. 1983, MNRAS, 202, 833

Rieke, G. H., \& Lebofsky, M. J. 1985, ApJ, 288, 618

Smith, B. J. 2003, AJ, 126, 935

Thackeray, A. D. 1977, Mem. R. Astr. Soc., 83, 1

Whitelock, P. A. 1987, PASP, 99, 573

Whitelock, P. A. 1988, in The Symbiotic Phenomenon, ed. M. Mikolajewski, et al. (Kluwer), 47

Whitelock, P. A. 1998, in Pulsating stars- Recent developments in Theory and Observation, ed. M. Takeuri, \& D. Sasselov (Universal Academy Press, Inc.), 31

Whitelock, P. A. 2003, in Symbiotic stars probing stellar evolution, ed. R. L. M. Corradi, J. Mikolajewska, \& T. J. Mahoney, ASP Conf. Ser., 303, 41

Whitelock, P. A., Feast, M. W., Catchpole, R. M., Carter, B. S., \& Roberts, G. 1983, MNRAS, 203, 351

Whitelock, P. A., Menzies, J., Feast, M. W., et al. 1994, MNRAS, 267, 711

Whitelock, P. A., Marang, F., \& Feast, M. W. 2000, MNRAS, 319, 728

Young, P. R., Berrington, K. A., \& Lobel, A. 2005, A\&A, 432, 665 